\title{
Development of a Comprehensive Analytical Method for Semi-Volatile Organic Compounds in Sediments by Using an Automated Identification and Quantification System with a GC-MS Database
}

\author{
Kiwao Kadokami, *† Shuangye Pan,* Duong Thi Hanh,* Xuehua LI,** and Terumi MiYazaki*** \\ *Faculty of Environmental Engineering, The University of Kitakyushu, 1-1 Hibikino, Wakamatsu, \\ Kitakyushu 808-0135, Japan \\ **Department of Environmental Science and Technology, Dalian University of Technology, Linggong Road 2, \\ Dalian 116024, China \\ ***Nippon Steel Kankyou Engineering Co., Ltd., 46-80 Nakabaru-sakinohama, Tobata, Kitakyushu 804-0002, \\ Japan
}

\begin{abstract}
A comprehensive analytical method for nearly 1000 semi-volatile organic compounds (SVOC) in sediments has been developed using an automated identification and quantification system with a GC-MS database. The results of recovery tests using model compounds, which comprise of 119 non-polar to polar compounds, showed that the method can quantitatively analyze most SVOC, except for very polar substances. Analytical results of a standard reference material were close to certified concentrations. The detection limits of the method were $4 \mu \mathrm{g} / \mathrm{kg}$ when measuring by TIM and $0.4 \mu \mathrm{g} / \mathrm{kg}$ by SIM. The method was applied to actual sediments in rivers in Ho Chi Minh City, Vietnam. A large number of substances, including persistent organic pollutants, which seem to be discharged from domestic sources, were found at relatively high concentrations. From these results, it is confirmed that the developed method is a useful way to obtain a holistic picture of pollution by SVOC, and is a good tool for rapid screening of chemical pollution in sediments.
\end{abstract}

(Received August 3, 2012; Accepted November 8, 2012; Published December 10, 2012)

\section{Introduction}

Although it is suspected that hundreds of thousands of chemical substances of anthropogenic origin exist in the environment, ${ }^{1}$ the number of chemicals that are regularly examined is very limited. In Japan, only 53 substances, the environmental standards and monitored substances, are monitored regularly in the aquatic environment, ${ }^{2}$ which is not enough to evaluate the safety of the environment and to protect aquatic life and human health; particularly important is to cope with pollution caused by accidents and natural disasters, such as earthquakes. In such cases it is necessary to analyze as many chemicals as possible as quickly as possible. Historically, when assessing large numbers of organic substances, many analytical methods have to be employed, which is time consuming and requires huge monetary expenditure. Preliminary screening of samples using rapid assessment tools is thus an increasingly attractive prospect for water environment managers. Consequently, we have developed an Automated Identification and Quantification System with a GC-MS Database (AIQS-DB) , $^{3,4}$ that can determine the concentrations of nearly 1000 semi-volatile organic compounds (SVOC). In addition, we have confirmed that the accuracy and precision of measurements using the AIQS-DB system are

† To whom correspondence should be addressed.

E-mail:kadokami@kitakyu-u.ac.jp almost the same as that obtained by the conventional internal standard methods (except for polar substances). ${ }^{5}$ Consequently, we are developing comprehensive analytical methods for various environmental substrates by making full use of the AIQS-DB. A combination of solid-phase extraction and the AIQS-DB was successfully developed for water samples except, again, for a range of polar substances. ${ }^{6}$ In the present study, we developed a comprehensive analytical method for sediment samples, and evaluated the performance and the effectiveness of the method by analyzing sediments collected in rivers in Ho Chi Minh City (HCM), Vietnam.

\section{Experimental}

Target compounds and reagents

The AIQS-DB consists of 914 SVOC (Table S1, Supporting Information). For the most part, these chemicals are regulated by the environmental protection laws in Japan or the United States, and were detected in an environmental survey conducted by the Japanese Ministry of the Environment (MOE). ${ }^{7}$ The 914 chemicals include almost all of the pesticides that can be currently measured by a GC, some pharmaceuticals and personal-care products (PPCPs), and some targeted groups, e.g. compounds eluted from tires, ${ }^{8}$ such as benzothiazoles. In a pretreatment procedure examination of liquid-liquid extraction (LLE), clean-up and overall recovery, we used 119 model 
compounds (MCs, Table 1) that are representatives of SVOC in the AIQS-DB, and consist of chemicals with wide range of physico-chemical properties. We also used 38 surrogates (deuterium-labeled internal standards, Table S2, Supporting Information) for examining any problems in analysis. The model compounds and surrogate compounds were obtained from Wako Pure Chemical (Osaka, Japan), Kanto Chemical (Tokyo, Japan), Hayashi Pure Chemical (Osaka, Japan), and Cambridge Isotope Laboratories, Inc. (Andover, MA, USA). Solvents were of pesticide residue analysis grade (Wako Pure Chemical, Japan).

\section{Analytical procedure}

Extraction from sediment was performed with an accelerated solvent extractor (ASE 350; Japan Dionex, Osaka, Japan). After mixing a sample (10 g, wet wt) with $7 \mathrm{~g}$ of Hydromatrix (Varian, Palo Alto, CA, USA), surrogates were spiked into the sample. The sample was put in an extraction vessel, and was then extracted with dichloromethane/acetone (1:1) for $30 \mathrm{~min}$ at $100^{\circ} \mathrm{C}$ at $10.3 \mathrm{MPa}$. Thereafter, the extract was concentrated to $10 \mathrm{ml}$ using a rotary evaporator, and then the concentrate was added to $200 \mathrm{ml}$ of a $5 \%$ sodium chloride solution. The solution was extracted with $30 \mathrm{ml}$ of dichloromethane twice. After dehydration of the extract with anhydrous sodium sulfate, the extract was concentrated to $10 \mathrm{ml}$. Thereafter, the solvent was changed from dichloromethane to hexane by adding $20 \mathrm{ml}$ of hexane to the concentrate and concentrating the resulting mixture to $1 \mathrm{ml}$ using a rotary evaporator. This procedure was carried out twice for a complete change of the solvent. The hexane concentrate obtained was applied to a silica-gel cartridge (Sep-Pak VAC $2 \mathrm{~g} / 12 \mathrm{ml}$; Waters Associates, Milford, MA, USA), and separated into 3 fractions by sequential elution with $15 \mathrm{ml}$ each of hexane (Fraction 1), 5\% acetone-hexane (Fraction 2), and 30\% acetone-hexane (Fraction 3). Fraction 1 was treated with copper powder (reduced copper, granular, super grade; Kishida Chemical, Tokyo, Japan) to remove sulfur. ${ }^{9}$ Fraction 3 was passed through an activated carbon column (ENVI-carb; Supelco, Bellefonte, PA, USA) to remove colored substances (e.g. non-volatile pigments) that damage a GC column. ${ }^{10}$ Each fraction was concentrated to $1 \mathrm{ml}$ with a rotary evaporator and a nitrogen stream, and then measured by GC-MS (QP-2010Plus; Shimadzu, Kyoto, Japan) after the addition of internal standards (Restek, Bellefonte, PA, USA). Each final test solution was measured by selected ion monitoring (SIM) and total ion monitoring (TIM) simultaneously. The GC-MS conditions for TIM were as described previously. ${ }^{3}$ SIM was applied to PCBs, organochlorine pesticides, polycyclic aromatic hydrocarbons (PAHs) and sterols; detailed SIM conditions are shown in Table S3 (Supporting Information).

\section{GC-MS analysis}

The chemical substances registered in the AIQS-DB (Compound composer, Shimadzu, Kyoto, Japan) were identified and quantified by using a combination of retention times, mass spectra, and internal standard calibration curves registered in the database. In order to obtain accurate results, a GC-MS has to be adjusted to designated conditions that are almost the same as the instrumental conditions when the database was constructed. The results obtained from performance check standards (Naginata criteria sample mix 3: Hayashi Pure Chemical, Osaka, Japan) are evaluated against three criteria: ${ }^{3,4}$ spectrum validity, inertness of column and inlet liner, and stability of response. When the results for performance check standards satisfy the criteria, the difference between the predicted and actual retention times is less than $3 \mathrm{~s}$, and obtained chemical concentrations (excluding some highly polar compounds which are difficult to measure by GC) are comparable to those obtained by conventional internal standard methods., ${ }^{4}$ However, in the present study to make sure of quantification, we quantified MCs in extraction tests by LLE, column chromatography and overall recovery tests by the conventional internal standard method instead of quantification by the AIQS-DB.

\section{Collection of sediment}

Sediment samples used in this study for evaluation of the performance of the developed method were collected from the surface layer of sediment at two sites (a small canal and the Saigon River) in HCM in February 2012 using an Eckman-Barge grab sampler $(15 \times 15 \mathrm{~cm})$.

\section{Results and Discussion}

Simultaneous analysis for a numerous compound with various physico-chemical properties is quite different from target analysis for a small number of compounds with similar properties. In the latter case, target analysis usually removes matrix and non-target substances as much as possible to improve the detection limits, precision and accuracy of analysis, whereas in the case of simultaneous analysis for a large number of substances, an extraction method should be selected that can extract as many compounds as possible, and its clean-up procedures are selected that remove the minimum substances necessary to prevent interference with the GC measurement. Therefore, we designed analytical procedures that are as simple as possible: extraction from sediment using a hydrophilic solvent, re-extraction with a hydrophobic solvent and adsorption chromatography to separate substances according to polarity.

Although there are several extraction methods for solid samples, such a Soxthlet or ultrasonic extraction, we chose an accelerated solvent extraction method because of its ability to extract a large number of compounds in a short period of time; the acetone/dichloromethane (1:1) mixture was used as the extraction solvent because this mixed solvent is recommended in US EPA Method 3545A. ${ }^{11}$ We selected dichloromethane as a solvent for re-extraction because dichloromethane is the most suitable solvent for wide spectrum SVOC. ${ }^{12-14}$ In adsorption chromatography, we examined three adsorbents: silica-gel, Florsil and alumina. The results of recovery of each pretreatment step, LLE with dichloromethane and column chromatography, and overall recovery through all the procedures, are described in the following sections.

\section{Recoveries of chemicals by liquid-liquid extraction with dichloromethane}

In the present study, LLE is one of the key analytical procedures because LLE is employed to extract SVOC after the extraction of sediments. We selected dichloromethane as an extraction solvent because it is usually used as a LLE solvent for SVOC, and its recoveries of SVOC have been well known. ${ }^{12-14}$ However, there has been no systematic examination on its extraction efficiency of SVOC based on the chemical physicochemical properties. In this context, we used $119 \mathrm{MCs}$ that were selected from the AIQS-DB as being representative of the SVOC in the AIQS-DB, and new chemicals that may be registered in the AIQS-DB in the future, to examine recovery by LLE. These MCs were chosen based on a compound category (e.g. see Table $\mathrm{S} 1$ ), and physico-chemical characteristics (such as boiling point, $\log K_{\text {ow }}$, polarity, presence of functional groups, active hydrogens). The extraction efficiencies were examined 
Table 1 Model compounds and their recoveries

\begin{tabular}{|c|c|c|c|c|c|c|c|c|c|c|c|c|}
\hline \multirow{3}{*}{ Compound } & \multirow{3}{*}{ Class $^{\mathrm{a}}$} & \multirow{3}{*}{$\begin{array}{l}\text { Molecular } \\
\text { formula }\end{array}$} & \multirow{3}{*}{$\mathrm{MW}^{\mathrm{b}}$} & \multirow{3}{*}{ CAS RNc } & \multirow{3}{*}{$\log K_{\text {ow }}{ }^{\mathrm{d}}$} & \multirow{2}{*}{\multicolumn{2}{|c|}{$\begin{array}{c}\text { Overall } \\
\text { recovery } \\
(n=7)\end{array}$}} & \multirow{3}{*}{$\begin{array}{c}\text { LLE } \\
\text { extraction }^{\mathrm{e}} \\
\text { efficiency } \\
(n=2), \%\end{array}$} & \multicolumn{4}{|c|}{ Silica-gel chromatography } \\
\hline & & & & & & & & & \multicolumn{3}{|c|}{ Elution pattern } & \multirow{2}{*}{$\begin{array}{c}\text { Recovery }{ }^{\mathrm{f}} \\
\%\end{array}$} \\
\hline & & & & & & $\%$ & RSD, \% & & Fr. 1 & Fr. 2 & Fr. 3 & \\
\hline 1 Flutolanil & 1 & $\mathrm{C}_{17} \mathrm{H}_{16} \mathrm{~F}_{3} \mathrm{NO}_{2}$ & 323 & $66332-96-5$ & 3.7 & 83 & 18 & 88 & & & $X$ & 102 \\
\hline $\begin{array}{l}2 \text { 1,1,1-Trichloro-2-methyl-2- } \\
\text { propanol }\end{array}$ & 2 & $\mathrm{C}_{4} \mathrm{H}_{7} \mathrm{Cl}_{3} \mathrm{O}$ & 176 & $57-15-8$ & 2.03 & 21 & 157 & 9 & & & $X$ & 94 \\
\hline 3 2-Ethyl-1-hexanol & 2 & $\mathrm{C}_{8} \mathrm{H}_{18} \mathrm{O}$ & 130 & $104-76-7$ & 2.73 & 116 & 127 & 116 & & $X$ & $\mathrm{X}$ & 119 \\
\hline 4 1-Octanol & 2 & $\mathrm{C}_{8} \mathrm{H}_{18} \mathrm{O}$ & 130 & $111-87-5$ & 3 & 124 & 23 & 82 & & & $\mathrm{X}$ & 118 \\
\hline 5 1-Nonanol & 2 & $\mathrm{C}_{9} \mathrm{H}_{20} \mathrm{O}$ & 144 & $143-08-8$ & 3.77 & 61 & 14 & 110 & & & $\mathrm{X}$ & 61 \\
\hline 6 3-Methoxy-1-butyl acetate & 3 & $\mathrm{C}_{7} \mathrm{H}_{14} \mathrm{O}_{3}$ & 146 & $4435-53-4$ & 1.01 & 113 & 7 & 76 & & $X$ & $X$ & 101 \\
\hline 7 Bis(2-ethylhexyl)sebacate & 3 & $\mathrm{C}_{26} \mathrm{H}_{50} \mathrm{O}_{4}$ & 426 & $122-62-3$ & 10.08 & 90 & 11 & 80 & & $X$ & & 101 \\
\hline $\begin{array}{l}8 \text { 3-Chloro-1,2- } \\
\text { dibromopropane }\end{array}$ & 4 & $\mathrm{C}_{3} \mathrm{H}_{5} \mathrm{Br}_{2} \mathrm{Cl}$ & 234 & $96-12-8$ & 2.96 & 77 & 10 & 71 & $X$ & $\mathrm{X}$ & & 94 \\
\hline $9 n$-Pentadecane & 4 & $\mathrm{C}_{15} \mathrm{H}_{32}$ & 212 & $629-62-9$ & 7.71 & 140 & 17 & 100 & $X$ & & & 143 \\
\hline $10 n$-Hexadecane & 4 & $\mathrm{C}_{16} \mathrm{H}_{34}$ & 226 & $544-76-3$ & 8.25 & 143 & 14 & 102 & $X$ & & & 196 \\
\hline $11 n$-Triacontane & 4 & $\mathrm{C}_{30} \mathrm{H}_{62}$ & 422 & $638-68-6$ & 15.07 & 119 & 9 & 89 & $X$ & & & 116 \\
\hline 12 Dibutylamine & 5 & $\mathrm{C}_{8} \mathrm{H}_{19} \mathrm{~N}$ & 129 & $111-92-2$ & 2.82 & 1 & 65 & 0 & & & & 0 \\
\hline 13 Caprolactam & 6 & $\mathrm{C}_{6} \mathrm{H}_{11} \mathrm{NO}$ & 113 & $105-60-2$ & 0.66 & 6 & 192 & 10 & & & $\mathrm{X}$ & 2 \\
\hline 14 Pyrimethanil & 7 & $\mathrm{C}_{12} \mathrm{H}_{13} \mathrm{~N}_{3}$ & 199 & $53112-28-0$ & 2.84 & 91 & 19 & 85 & & $X$ & $X$ & 96 \\
\hline 15 Aniline & 8 & $\mathrm{C}_{6} \mathrm{H}_{7} \mathrm{~N}$ & 93 & $62-53-3$ & 0.94 & 39 & 36 & 106 & & & $X$ & 34 \\
\hline 16 2,6-Dimethylaniline & 8 & $\mathrm{C}_{8} \mathrm{H}_{11} \mathrm{~N}$ & 121 & $87-62-7$ & 1.84 & 70 & 27 & 81 & & $X$ & $\mathrm{X}$ & 128 \\
\hline 17 3,4-Dimethylaniline & 8 & $\mathrm{C}_{8} \mathrm{H}_{11} \mathrm{~N}$ & 121 & $95-64-7$ & 1.84 & 34 & 58 & 101 & & $X$ & $X$ & 59 \\
\hline $18 m$-Aminophenol & 8 & $\mathrm{C}_{6} \mathrm{H}_{7} \mathrm{NO}$ & 109 & $591-27-5$ & 0.21 & 1 & 155 & 0 & & & $X$ & 2 \\
\hline $19 p$-Phenylenediamine & 8 & $\mathrm{C}_{6} \mathrm{H}_{8} \mathrm{~N}_{2}$ & 108 & $106-50-3$ & -0.3 & 11 & 38 & 59 & & & $\mathrm{X}$ & 23 \\
\hline $20 \mathrm{~m}$-Phenylenediamine & 8 & $\mathrm{C}_{6} \mathrm{H}_{8} \mathrm{~N}_{2}$ & 108 & $108-45-2$ & -0.33 & 11 & 38 & 66 & & & $X$ & 52 \\
\hline 21 2-Amino-5-chlorotoluene & 8 & $\mathrm{C}_{7} \mathrm{H}_{8} \mathrm{NCl}$ & 141 & $95-69-2$ & 2.27 & 74 & 20 & 79 & & & $X$ & 93 \\
\hline 22 2,4-Dichloroaniline & 8 & $\mathrm{C}_{6} \mathrm{H}_{5} \mathrm{NCl}_{2}$ & 161 & $554-00-7$ & 2.78 & 112 & 20 & 94 & & $X$ & $X$ & 94 \\
\hline 23 2,6-Diaminotoluene & 8 & $\mathrm{C}_{7} \mathrm{H}_{10} \mathrm{~N}_{2}$ & 122 & $823-40-5$ & 0.16 & 18 & 65 & 41 & & & $X$ & 51 \\
\hline $\begin{array}{l}24 \mathrm{~N} \text {-Nitrosodiphenylamine } \\
\text { (DPA) }\end{array}$ & 8 & $\mathrm{C}_{12} \mathrm{H}_{10} \mathrm{~N}_{2} \mathrm{O}$ & 198 & $86-30-6$ & 3.13 & 114 & 12 & 96 & & $\mathrm{X}$ & & 88 \\
\hline 25 2,4-Dinitroaniline & 8 & $\mathrm{C}_{6} \mathrm{H}_{5} \mathrm{~N}_{3} \mathrm{O}_{4}$ & 183 & $97-02-9$ & 1.84 & 33 & 41 & 91 & & & $X$ & 50 \\
\hline 26 Benzidine & 8 & $\mathrm{C}_{12} \mathrm{H}_{12} \mathrm{~N}_{2}$ & 184 & $92-87-5$ & 1.34 & 1 & 42 & 106 & & & $X$ & 16 \\
\hline $27 \mathrm{~N}$-Phenyl-1-naphthylamine & 8 & $\mathrm{C}_{16} \mathrm{H}_{13} \mathrm{~N}$ & 219 & $90-30-2$ & 4.2 & 63 & 22 & 88 & & $X$ & & 97 \\
\hline 28 3,3'-Dichlorobenzidine & 8 & $\mathrm{C}_{12} \mathrm{H}_{10} \mathrm{~N}_{2} \mathrm{Cl}_{2}$ & 252 & $91-94-1$ & 3.51 & 19 & 119 & 72 & & & $\mathrm{X}$ & 72 \\
\hline $\begin{array}{l}29 \text { 4,4'-Methylenebis }(2- \\
\text { chloroaniline })\end{array}$ & 8 & $\mathrm{C}_{13} \mathrm{H}_{12} \mathrm{~N}_{2} \mathrm{C}_{12}$ & 266 & $101-14-4$ & 3.91 & 62 & 49 & 87 & & & $X$ & 84 \\
\hline 30 Isoprothiolane & 9 & $\mathrm{C}_{12} \mathrm{H}_{18} \mathrm{O}_{4} \mathrm{~S}_{2}$ & 290 & $50512-35-1$ & 2.88 & 76 & 16 & 91 & & & $X$ & 109 \\
\hline 31 Benzyl chloride & 10 & $\mathrm{C}_{7} \mathrm{H}_{7} \mathrm{Cl}$ & 126 & $100-44-7$ & 2.3 & 74 & 10 & 103 & $\mathrm{X}$ & & & 87 \\
\hline 32 Benzyl alcohol & 10 & $\mathrm{C}_{7} \mathrm{H}_{8} \mathrm{O}$ & 108 & $100-51-6$ & 1.1 & 39 & 28 & 102 & & & $X$ & 47 \\
\hline 33 Hexachlorobenzene & 10 & $\mathrm{C}_{6} \mathrm{C}_{16}$ & 282 & 118-74-1 & 5.73 & 86 & 10 & 94 & $\mathrm{X}$ & & & 89 \\
\hline 34 1,2,4,5-Tetrabromobenzene & 10 & $\mathrm{C}_{6} \mathrm{H}_{2} \mathrm{Br}_{4}$ & 390 & $636-28-2$ & 5.13 & 92 & 13 & 95 & $X$ & & & 97 \\
\hline 35 Triphenylmethane & 10 & $\mathrm{C}_{19} \mathrm{H}_{16}$ & 244 & $519-73-3$ & 5.37 & 89 & 11 & 86 & & $X$ & & 98 \\
\hline 36 Biphenyl & 11 & $\mathrm{C}_{12} \mathrm{H}_{10}$ & 154 & $92-52-4$ & 3.98 & 85 & 10 & 98 & $X$ & & & 96 \\
\hline $\begin{array}{l}372,2^{\prime}, 4,4^{\prime}, 5,5^{\prime} \text {-Hexabromo- } \\
\text { biphenyl }\end{array}$ & 11 & $\mathrm{C}_{12} \mathrm{H}_{4} \mathrm{Br}_{6}$ & 622 & $59080-40-9$ & - & 97 & 8 & 94 & $X$ & & & 100 \\
\hline 38 Methyl dymron & 12 & $\mathrm{C}_{17} \mathrm{H}_{20} \mathrm{~N}_{2} \mathrm{O}$ & 268 & $42609-73-4$ & 3.01 & 70 & 26 & 93 & & & $X$ & 92 \\
\hline 39 Carbofuran & 13 & $\mathrm{C}_{12} \mathrm{H}_{15} \mathrm{NO}_{3}$ & 221 & $1563-66-2$ & 2.32 & 75 & 21 & 82 & & & $\mathrm{X}$ & 90 \\
\hline 40 Thiobencarb & 13 & $\mathrm{C}_{12} \mathrm{H}_{16} \mathrm{NOSCl}$ & 257 & 28249-77-6 & 3.4 & 91 & 13 & 101 & & $\mathrm{X}$ & & 102 \\
\hline 41 Tebufenpyrad & 14 & $\mathrm{C}_{18} \mathrm{H}_{24} \mathrm{ClN}_{3} \mathrm{O}$ & 333 & $119168-77-3$ & 4.61 & 95 & 16 & 84 & & $X$ & $X$ & 105 \\
\hline 42 Iprodione & 15 & $\mathrm{C}_{13} \mathrm{H}_{13} \mathrm{Cl}_{2} \mathrm{~N}_{3} \mathrm{O}_{3}$ & 329 & $36734-19-7$ & 3 & 85 & 22 & 86 & & & $X$ & 89 \\
\hline 43 Trifluralin & 16 & $\mathrm{C}_{13} \mathrm{H}_{16} \mathrm{~F}_{3} \mathrm{~N}_{3} \mathrm{O}_{4}$ & 335 & $1582-09-8$ & 5.34 & 88 & 13 & 79 & & $X$ & & 82 \\
\hline 44 Benfluralin & 16 & $\mathrm{C}_{13} \mathrm{H}_{16} \mathrm{~F}_{3} \mathrm{~N}_{3} \mathrm{O}_{4}$ & 335 & $1861-40-1$ & 5.29 & 114 & 12 & 80 & & $X$ & & 79 \\
\hline 45 Triclosan & 17 & $\mathrm{C}_{12} \mathrm{H}_{7} \mathrm{O}_{2} \mathrm{Cl}_{3}$ & 288 & $3380-34-5$ & 4.76 & 82 & 17 & 72 & & $\mathrm{X}$ & $X$ & 84 \\
\hline 46 Pyriproxyfen & 17 & $\mathrm{C}_{20} \mathrm{H}_{19} \mathrm{NO}_{3}$ & 321 & $95737-68-1$ & 5.55 & 82 & 22 & 91 & & $\mathrm{X}$ & & 94 \\
\hline 47 Bis(2-chloroethyl)ether & 18 & $\mathrm{C}_{4} \mathrm{H}_{8} \mathrm{Cl}_{2} \mathrm{O}$ & 142 & $111-44-4$ & 1.29 & 79 & 7 & 97 & & $\mathrm{X}$ & $\mathrm{X}$ & 103 \\
\hline 48 Benzothiazole & 19 & $\mathrm{C}_{7} \mathrm{H}_{5} \mathrm{NS}$ & 135 & $95-16-9$ & 2.01 & 80 & 13 & 94 & & $\mathrm{X}$ & & 99 \\
\hline 49 Dibenzothiophene & 19 & $\mathrm{C}_{12} \mathrm{H}_{8} \mathrm{~S}$ & 184 & $132-65-0$ & 4.38 & 89 & 9 & 94 & $\mathrm{X}$ & & & 93 \\
\hline 50 Bentazone & 20 & $\mathrm{C}_{10} \mathrm{H}_{12} \mathrm{~N}_{2} \mathrm{O}_{3} \mathrm{~S}$ & 240 & $25057-89-0$ & -0.49 & 0 & 59 & 1 & & & $\mathrm{X}$ & 4 \\
\hline 51 Tricyclazole & 21 & $\mathrm{C}_{9} \mathrm{H}_{7} \mathrm{~N}_{3} \mathrm{~S}$ & 189 & 41814-78-2 & 1.42 & 14 & 118 & 92 & & & $\mathrm{X}$ & 18 \\
\hline 52 Nitrobenzene & 22 & $\mathrm{C}_{6} \mathrm{H}_{5} \mathrm{NO}_{2}$ & 123 & $98-95-3$ & 1.85 & 77 & 7 & 92 & & $\mathrm{X}$ & & 97 \\
\hline 53 3-Chloronitrobenzene & 22 & $\mathrm{C}_{6} \mathrm{H}_{4} \mathrm{NO}_{2} \mathrm{Cl}$ & 157 & $100-00-5$ & 2.39 & 78 & 3 & 76 & & $\mathrm{X}$ & & 88 \\
\hline
\end{tabular}




\begin{tabular}{|c|c|c|c|c|c|c|c|c|c|c|c|c|}
\hline \multirow{3}{*}{ Compound } & \multirow{3}{*}{ Class $^{\mathrm{a}}$} & \multirow{3}{*}{$\begin{array}{l}\text { Molecular } \\
\text { formula }\end{array}$} & \multirow{3}{*}{$\mathrm{MW}^{\mathrm{b}}$} & \multirow{3}{*}{$\mathrm{CAS} \mathrm{RN}^{\mathrm{c}}$} & \multirow{3}{*}{$\log K_{\text {ow }}{ }^{d}$} & \multirow{2}{*}{\multicolumn{2}{|c|}{$\begin{array}{c}\text { Overall } \\
\text { recovery } \\
(n=7)\end{array}$}} & \multirow{3}{*}{$\begin{array}{c}\text { LLE } \\
\text { extraction } \\
\text { efficiency }^{\text {e }} \\
(n=2), \%\end{array}$} & \multicolumn{4}{|c|}{ Silica-gel chromatography } \\
\hline & & & & & & & & & \multicolumn{3}{|c|}{ Elution pattern } & \multirow{2}{*}{$\begin{array}{c}\text { Recovery, }{ }^{\mathrm{f}} \\
\%\end{array}$} \\
\hline & & & & & & $\%$ & $\mathrm{RSD}, \%$ & & Fr. 1 & Fr. 2 & Fr. 3 & \\
\hline 54 2,4-Dichloronitrobenzene & 22 & $\mathrm{C}_{6} \mathrm{H}_{3} \mathrm{NO}_{2} \mathrm{Cl}_{2}$ & 191 & $611-06-3$ & 3.07 & 108 & 9 & 87 & & $\mathrm{X}$ & & 95 \\
\hline 55 2,4,6-Trinitrotoluene & 22 & $\mathrm{C}_{7} \mathrm{H}_{5} \mathrm{~N}_{3} \mathrm{O}_{6}$ & 227 & $118-96-7$ & 1.6 & 96 & 16 & 87 & & $X$ & $\mathrm{X}$ & 82 \\
\hline 56 Pentachloronitrobenzene & 22 & $\mathrm{C}_{6} \mathrm{NO}_{2} \mathrm{Cl}_{5}$ & 293 & $82-68-8$ & 4.64 & 84 & 11 & 87 & $\mathrm{X}$ & & & 84 \\
\hline 57 2-Nitrophenol & 23 & $\mathrm{C}_{6} \mathrm{H}_{5} \mathrm{NO}_{3}$ & 139 & $88-75-5$ & 1.79 & 69 & 15 & 81 & & $\mathrm{X}$ & & 90 \\
\hline 58 4-Nitrophenol & 23 & $\mathrm{C}_{6} \mathrm{H}_{5} \mathrm{NO}_{3}$ & 139 & $100-02-7$ & 1.91 & 46 & 35 & 59 & & & $\mathrm{X}$ & 64 \\
\hline 59 4-Methyl-3-nitrophenol & 23 & $\mathrm{C}_{7} \mathrm{H}_{7} \mathrm{NO}_{3}$ & 153 & $2042-14-0$ & 2.18 & 76 & 27 & 60 & & & $\mathrm{X}$ & 64 \\
\hline 60 1-Nitronaphthalene & 24 & $\mathrm{C}_{10} \mathrm{H}_{7} \mathrm{NO}_{2}$ & 173 & $86-57-7$ & 3.19 & 110 & 10 & 93 & & $\mathrm{X}$ & $\mathrm{X}$ & 79 \\
\hline $61 \alpha-\mathrm{HCH}$ & 25 & $\mathrm{C}_{6} \mathrm{H}_{6} \mathrm{Cl}_{6}$ & 288 & $319-84-6$ & 3.8 & 93 & 11 & 91 & & $X$ & & 97 \\
\hline $62 \beta-\mathrm{HCH}$ & 25 & $\mathrm{C}_{6} \mathrm{H}_{6} \mathrm{Cl}_{6}$ & 288 & $319-85-7$ & 3.78 & 99 & 9 & 91 & & $\mathrm{X}$ & & 99 \\
\hline $63 \delta-\mathrm{HCH}$ & 25 & $\mathrm{C}_{6} \mathrm{H}_{6} \mathrm{Cl}_{6}$ & 288 & $58-89-9$ & 3.72 & 99 & 8 & 88 & & $X$ & & 94 \\
\hline $64 \mathrm{~d}-\mathrm{HCH}$ & 25 & $\mathrm{C}_{6} \mathrm{H}_{6} \mathrm{Cl}_{6}$ & 288 & $319-86-8$ & 4.14 & 100 & 10 & 93 & & $X$ & & 102 \\
\hline 65 Heptachlor & 25 & $\mathrm{C}_{10} \mathrm{H}_{5} \mathrm{Cl}_{7}$ & 370 & $76-44-8$ & 6.1 & 97 & 12 & 66 & $\mathrm{X}$ & & & 82 \\
\hline 66 Aldrin & 25 & $\mathrm{C}_{12} \mathrm{H}_{8} \mathrm{Cl}_{6}$ & 362 & $309-00-2$ & 6.5 & 92 & 9 & 83 & $X$ & & & 108 \\
\hline 67 Heptachlor epoxide & 25 & $\mathrm{C}_{10} \mathrm{H}_{5} \mathrm{Cl}_{7} \mathrm{O}$ & 386 & $1024-57-3$ & 4.98 & 92 & 11 & 93 & & $\mathrm{X}$ & & 83 \\
\hline 68 Captan & 25 & $\mathrm{C}_{9} \mathrm{H}_{8} \mathrm{Cl}_{3} \mathrm{NO}_{2} \mathrm{~S}$ & 299 & $133-06-2$ & 2.8 & 100 & 17 & 106 & & & $\mathrm{X}$ & 136 \\
\hline 69 trans-Nonachlor & 25 & $\mathrm{C}_{10} \mathrm{H}_{5} \mathrm{Cl}_{9}$ & 440 & $39765-80-5$ & 6.35 & 89 & 11 & 93 & $X$ & & & 84 \\
\hline 70 Dieldrin & 25 & $\mathrm{C}_{12} \mathrm{H}_{8} \mathrm{Cl}_{6} \mathrm{O}$ & 378 & $60-57-1$ & 5.4 & 98 & 11 & 104 & & $\mathrm{X}$ & & 100 \\
\hline $71 p, p^{\prime}-\mathrm{DDE}$ & 25 & $\mathrm{C}_{14} \mathrm{H}_{8} \mathrm{Cl}_{4}$ & 316 & $72-55-9$ & 6.51 & 89 & 7 & 86 & $\mathrm{X}$ & & & 108 \\
\hline 72 Endrin & 25 & $\mathrm{C}_{12} \mathrm{H}_{8} \mathrm{Cl}_{6} \mathrm{O}$ & 378 & $72-20-8$ & 5.2 & 100 & 13 & 82 & & $X$ & & 85 \\
\hline $73 p, p^{\prime}$-DDD & 25 & $\mathrm{C}_{14} \mathrm{H}_{10} \mathrm{Cl}_{4}$ & 318 & $72-54-8$ & 6.02 & 81 & 14 & 89 & $\mathrm{X}$ & $\mathrm{X}$ & & 107 \\
\hline $74 o, p^{\prime}$-DDT & 25 & $\mathrm{C}_{14} \mathrm{H}_{9} \mathrm{Cl}_{5}$ & 352 & $789-02-6$ & 6.79 & 103 & 10 & 92 & $X$ & & & 98 \\
\hline $75 p, p^{\prime}$-DDT & 25 & $\mathrm{C}_{14} \mathrm{H}_{9} \mathrm{Cl}_{5}$ & 352 & $50-29-3$ & 6.91 & 108 & 11 & 92 & $\mathrm{X}$ & & & 93 \\
\hline 76 Phenol & 26 & $\mathrm{C}_{6} \mathrm{H}_{6} \mathrm{O}$ & 94 & $108-95-2$ & 1.46 & 32 & 21 & 70 & & & $\mathrm{X}$ & 111 \\
\hline 77 Diazinon & 26 & $\mathrm{C}_{12} \mathrm{H}_{21} \mathrm{~N}_{2} \mathrm{O}_{3} \mathrm{PS}$ & 304 & $333-41-5$ & 3.81 & 88 & 12 & 97 & & $X$ & $\mathrm{X}$ & 106 \\
\hline 78 Isoxathion & 26 & $\mathrm{C}_{13} \mathrm{H}_{16} \mathrm{NO}_{4} \mathrm{PS}$ & 313 & 18854-01-8 & 3.73 & 119 & 12 & 100 & & $\mathrm{X}$ & & 109 \\
\hline 79 Edifenphos & 26 & $\mathrm{C}_{14} \mathrm{H}_{15} \mathrm{O}_{2} \mathrm{PS}_{2}$ & 310 & $17109-49-8$ & 3.48 & 90 & 23 & 80 & & & $\mathrm{X}$ & 105 \\
\hline 80 EPN & 26 & $\mathrm{C}_{14} \mathrm{H}_{14} \mathrm{NO}_{4} \mathrm{PS}$ & 323 & $2104-64-5$ & 4.78 & 91 & 13 & 112 & & $X$ & & 95 \\
\hline 81 2-Chloro-6-methylphenol & 27 & $\mathrm{C}_{7} \mathrm{H}_{7} \mathrm{OCl}$ & 142 & $87-64-9$ & 2.8 & 83 & 11 & 96 & & $X$ & & 94 \\
\hline 82 2,6-Dimethylphenol & 27 & $\mathrm{C}_{8} \mathrm{H}_{10} \mathrm{O}$ & 122 & $576-26-1$ & 2.36 & 85 & 10 & 81 & & $\mathrm{X}$ & $\mathrm{X}$ & 102 \\
\hline 83 3-Chlorophenol & 27 & $\mathrm{C}_{6} \mathrm{H}_{5} \mathrm{OCl}$ & 128 & $108-43-0$ & 2.5 & 88 & 7 & 78 & & & $\mathrm{X}$ & 96 \\
\hline 84 2,6-Dichlorophenol & 27 & $\mathrm{C}_{6} \mathrm{H}_{4} \mathrm{OCl}_{2}$ & 162 & $87-65-0$ & 2.75 & 77 & 11 & 90 & & $\mathrm{X}$ & $\mathrm{X}$ & 90 \\
\hline 85 1,4-Benzenediol & 27 & $\mathrm{C}_{6} \mathrm{H}_{6} \mathrm{O}_{2}$ & 110 & $123-31-9$ & 0.59 & 14 & 52 & 2 & & & $\mathrm{X}$ & 2 \\
\hline 86 4-tert-Butylphenol & 27 & $\mathrm{C}_{10} \mathrm{H}_{14} \mathrm{O}$ & 150 & $98-54-4$ & 3.31 & 88 & 15 & 86 & & & $\mathrm{X}$ & 93 \\
\hline 87 2,4,6-Trichlorophenol & 27 & $\mathrm{C}_{6} \mathrm{H}_{3} \mathrm{OCl}_{3}$ & 196 & $88-06-2$ & 3.69 & 76 & 13 & 89 & & $\mathrm{X}$ & $\mathrm{X}$ & 100 \\
\hline 88 Pentachlorophenol & 27 & $\mathrm{C}_{6} \mathrm{HOCl}_{5}$ & 266 & $87-86-5$ & 5.01 & 9 & 77 & 41 & & & $\mathrm{X}$ & 68 \\
\hline 89 4-n-Nonylphenol & 27 & $\mathrm{C}_{15} \mathrm{H}_{24} \mathrm{O}$ & 220 & $104-40-5$ & 5.76 & 332 & 34 & 90 & & & $\mathrm{X}$ & 612 \\
\hline 90 Bisphenol A & 27 & $\mathrm{C}_{15} \mathrm{H}_{16} \mathrm{O}_{2}$ & 228 & $80-05-7$ & 3.32 & 53 & 42 & 110 & & & $\mathrm{X}$ & 22 \\
\hline 91 Tris(2-chloroethyl)phosphate & 28 & $\mathrm{C}_{6} \mathrm{H}_{12} \mathrm{O}_{4} \mathrm{PCl}_{3}$ & 284 & $115-96-8$ & 1.78 & 84 & 7 & 106 & & & $\mathrm{X}$ & 102 \\
\hline 92 Tributyl phosphate & 28 & $\mathrm{C}_{12} \mathrm{H}_{27} \mathrm{O}_{4} \mathrm{P}$ & 266 & $126-73-8$ & 4.0 & 94 & 27 & 92 & & & $\mathrm{X}$ & 97 \\
\hline 93 Tris(2-ethylhexyl)phosphate & 28 & $\mathrm{C}_{24} \mathrm{H}_{51} \mathrm{O}_{4} \mathrm{P}$ & 434 & $78-42-2$ & 9.49 & 92 & 15 & 108 & & $\mathrm{X}$ & $\mathrm{X}$ & 93 \\
\hline 94 Dimethyl phthalate & 29 & $\mathrm{C}_{10} \mathrm{H}_{10} \mathrm{O}_{4}$ & 194 & $131-11-3$ & 1.6 & 102 & 14 & 87 & & $\mathrm{X}$ & $\mathrm{X}$ & 66 \\
\hline 95 Dimethyl terephthalate & 29 & $\mathrm{C}_{10} \mathrm{H}_{10} \mathrm{O}_{4}$ & 194 & $120-61-6$ & 2.25 & 108 & 13 & 101 & & $\mathrm{X}$ & & 98 \\
\hline 96 Naphthalene & 30 & $\mathrm{C}_{10} \mathrm{H}_{8}$ & 128 & $91-20-3$ & 3.3 & 85 & 8 & 89 & $X$ & & & 91 \\
\hline 97 Acenaphthylene & 30 & $\mathrm{C}_{12} \mathrm{H}_{8}$ & 152 & $208-96-8$ & 3.94 & 85 & 10 & 94 & $\mathrm{X}$ & & & 101 \\
\hline 98 Acenaphthene & 30 & $\mathrm{C}_{12} \mathrm{H}_{10}$ & 154 & $83-32-9$ & 3.92 & 115 & 12 & 95 & $\mathrm{X}$ & & & 106 \\
\hline 99 Fluorene & 30 & $\mathrm{C}_{13} \mathrm{H}_{10}$ & 166 & $86-73-7$ & 4.18 & 111 & 11 & 98 & $\mathrm{X}$ & & & 101 \\
\hline 100 Phenanthrene & 30 & $\mathrm{C}_{14} \mathrm{H}_{10}$ & 178 & $85-01-8$ & 4.46 & 115 & 13 & 93 & $\mathrm{X}$ & & & 105 \\
\hline 101 Anthracene & 30 & $\mathrm{C}_{14} \mathrm{H}_{10}$ & 178 & $120-12-7$ & 4.45 & 88 & 10 & 92 & $\mathrm{X}$ & & & 101 \\
\hline 102 Fluoranthene & 30 & $\mathrm{C}_{16} \mathrm{H}_{10}$ & 202 & $206-44-0$ & 5.16 & 106 & 15 & 93 & $\mathrm{X}$ & $X$ & & 95 \\
\hline 103 Pyrene & 30 & $\mathrm{C}_{16} \mathrm{H}_{10}$ & 202 & $129-00-0$ & 4.88 & 97 & 17 & 100 & $\mathrm{X}$ & & & 99 \\
\hline $104 \operatorname{Benz}(a)$ anthracene & 30 & $\mathrm{C}_{18} \mathrm{H}_{12}$ & 228 & $56-55-3$ & 5.76 & 90 & 13 & 108 & $\mathrm{X}$ & $X$ & & 95 \\
\hline 105 Chrysene & 30 & $\mathrm{C}_{18} \mathrm{H}_{12}$ & 228 & $218-01-9$ & 5.81 & 92 & 12 & 108 & $X$ & $\mathrm{X}$ & & 90 \\
\hline $106 \operatorname{Benzo}(k)$ fluoranthene & 30 & $\mathrm{C}_{20} \mathrm{H}_{12}$ & 252 & $207-08-9$ & 6.11 & 91 & 16 & 98 & & $\mathrm{X}$ & & 89 \\
\hline 107 Benzo(b)fluoranthene & 30 & $\mathrm{C}_{20} \mathrm{H}_{12}$ & 252 & $205-99-2$ & 5.78 & 90 & 12 & 97 & & $X$ & & 84 \\
\hline $108 \operatorname{Benzo}(e)$ pyrene & 30 & $\mathrm{C}_{20} \mathrm{H}_{12}$ & 252 & $192-97-2$ & 6.44 & 90 & 10 & 95 & $X$ & $X$ & & 86 \\
\hline $109 \operatorname{Benzo}(a)$ pyrene & 30 & $\mathrm{C}_{20} \mathrm{H}_{12}$ & 252 & $50-32-8$ & 6.13 & 90 & 12 & 92 & $X$ & $\mathrm{X}$ & & 85 \\
\hline 110 Indeno(1,2,3-cd)pyrene & 30 & $\mathrm{C}_{22} \mathrm{H}_{12}$ & 276 & $193-39-5$ & 6.7 & 96 & 14 & 95 & & $\mathrm{X}$ & & 88 \\
\hline 111 Dibenz $(a, h)$ anthracene & 30 & $\mathrm{C}_{22} \mathrm{H}_{14}$ & 278 & $53-70-3$ & 6.75 & 65 & 17 & 82 & & $\mathrm{X}$ & & 79 \\
\hline $112 \operatorname{Benzo}(g h i)$ perylene & 30 & $\mathrm{C}_{22} \mathrm{H}_{12}$ & 276 & $191-24-2$ & 6.63 & 97 & 12 & 99 & & $\mathrm{X}$ & & 90 \\
\hline
\end{tabular}




\begin{tabular}{|c|c|c|c|c|c|c|c|c|c|c|c|c|}
\hline \multirow{3}{*}{ No. } & \multirow{3}{*}{ Compound } & \multirow{3}{*}{ Class $^{\mathrm{a}}$} & \multirow{3}{*}{$\begin{array}{l}\text { Molecular } \\
\text { formula }\end{array}$} & \multirow{3}{*}{$\mathrm{MW}^{\mathrm{b}}$} & \multirow{3}{*}{ CAS RN ${ }^{c}$} & \multirow{3}{*}{$\log K_{\text {ow }}{ }^{\mathrm{d}}$} & \multirow{2}{*}{\multicolumn{2}{|c|}{$\begin{array}{l}\text { Overall } \\
\text { recovery } \\
(n=7)\end{array}$}} & \multirow{3}{*}{$\begin{array}{c}\text { LLE } \\
\text { extraction } \\
\text { efficiency }^{\mathrm{e}} \\
(n=2), \%\end{array}$} & \multicolumn{3}{|c|}{ Silica-gel chromatography } \\
\hline & & & & & & & & & & \multicolumn{2}{|c|}{ Elution pattern } & \multirow{2}{*}{$\underset{\%}{\text { Recovery }}{ }^{\mathrm{f}}$} \\
\hline & & & & & & & $\%$ & $\mathrm{RSD}, \%$ & & Fr. 1 Fr. 2 & Fr. 3 & \\
\hline $113 \mathrm{~T}$ & & 31 & $\mathrm{C}_{17} \mathrm{H}_{14} \mathrm{ClF}_{7} \mathrm{O}_{2}$ & 418 & $79538-32-2$ & 6.5 & 97 & 10 & 88 & $\mathrm{X}$ & & 103 \\
\hline $114 \mathrm{E}$ & ezin & 32 & $\mathrm{C}_{16} \mathrm{H}_{23} \mathrm{~N}_{3} \mathrm{OS}$ & 305 & $69327-76-0$ & 4.3 & 87 & 15 & 94 & $\mathrm{X}$ & $\mathrm{X}$ & 106 \\
\hline $115 n$ & henyl & 33 & $\mathrm{C}_{18} \mathrm{H}_{14}$ & 230 & $92-06-8$ & 5.52 & 92 & 9 & 93 & $\mathrm{X}$ & & 101 \\
\hline $116 p$ & enyl & 33 & $\mathrm{C}_{18} \mathrm{H}_{14}$ & 230 & $92-94-4$ & 5.6 & 92 & 10 & 91 & $\mathrm{X}$ & & 100 \\
\hline $117 \mathrm{~A}$ & & 34 & $\mathrm{C}_{8} \mathrm{H}_{14} \mathrm{~N}_{5} \mathrm{Cl}$ & 215 & $1912-24-9$ & 2.61 & 79 & 14 & 82 & & $\mathrm{X}$ & 103 \\
\hline $118 \mathrm{~s}$ & & 34 & $\mathrm{C}_{8} \mathrm{H}_{15} \mathrm{~N}_{5} \mathrm{~S}$ & 213 & $1014-70-6$ & 2.6 & 52 & 19 & 69 & & $\mathrm{X}$ & 99 \\
\hline $119 \mathrm{~T}$ & nazole & 35 & $\mathrm{C}_{13} \mathrm{H}_{11} \mathrm{Cl}_{2} \mathrm{~F}_{4} \mathrm{~N}_{3} \mathrm{O}$ & 371 & $112281-77-3$ & 3.56 & 71 & 22 & 92 & & $\mathrm{X}$ & 82 \\
\hline
\end{tabular}

a. 1, Acid amide pesticides; 2, alcohols; 3 , aliphatic esters; 4, alkanes; 5, amines; 6 , lactam; 7, anilinopyrimidine pesticide; 8 , aromatic amines; 9 , aromatic carbonic acid pesticides; 10 , benzenes; 11, biphenyls; 12 , carbaimide pesticides; 13 , carbamate pesticides; 14 , diazole pesticides; 15, dicarboxyimide pesticides; 16, dinitroaniline pesticides; 17, diphenylether pesticides; 18, ethers; 19, heterocyclic aromatic compounds; 20, benzothiadiazinone; 21, heterocyclic pesticides; 22, nitrobenzenes; 23, nitrophenols; 24, nitropolycyclic aromatic hydrocarbons; 25, organochlorine pesticides; 26, organophosphorus pesticides; 27 , phenols; 28, phosphates; 29, phthalates; 30, polycyclic aromatic hydrocarbons; 31 , pyrethroid pesticides; 32, thiazinone pesticides; 33, terphenyls; 34, triazine pesticides; 35 , triazole pesticides. b. Molecular weight. c. Chemical Abstracts registry number. d. Logarithm of octanol-water partition coefficient. e. Liquid-liquid extraction with dichloromethane. f. No subtraction of blank values.

by adding each $2 \mu \mathrm{g}$ of $\mathrm{MCs}$ to $200 \mathrm{~mL}$ of $5 \% \mathrm{NaCl}$ water. Quantification was done by the internal standard method. Compounds whose octanol-water partition coefficient $\left(\log K_{\text {ow }}\right)$ are above 1 show more than 60\% of recovery (Table 1 ). However, compounds that dissociate in water, such as pentachlorophenol, had low recovery, even though they may have a $\log K_{\text {ow }}>1$. In addition, compounds whose $\log K_{\text {ow }}$ are low and also have more than one polar functional group also showed low recovery: e.g. 1,4-benzenediol and 1,1,1-trichloro-2-methyl-2-propanol. The substances with low recovery were consistent with substances that are difficult to measure by the GC-MS conditions ${ }^{5}$ used in the present study. Consequently, it was confirmed that SVOC in the AIQS-DB can be extracted by LLE with dichloromethane, except for a number of highly polar substances.

Examination of clean-up by adsorption chromatography with silica-gel

Since sediments usually contain a large amount of organic matrix and sulfides, a clean-up procedure is essential. In the present study, we adopted adsorption chromatography to separate SVOC according to their polarities and copper treatment to remove sulfur. ${ }^{9}$ Although the adopted clean-up procedure cannot precisely separate chemicals, it seems to be suitable for comprehensive analysis, because our large number of target compounds have a wide range of physico-chemical properties. In the present study, we tested three types of adsorbents: silica-gel (Sep-Pak VAC 2 g/12 ml; Waters Associates, Milford, MA, USA), Florsil (Waters Sep-Pac VAC $2 \mathrm{~g}$ ) and neutral alumina (Waters Sep-Pac VAC $2 \mathrm{~g}$ ). We selected a hexane-acetone mixture as elution solvents: 1st fraction, hexane, 2nd fraction, 5\% acetone-hexane and 3rd fraction, 30\% acetone-hexane; elution volumes are $15 \mathrm{ml}$ each. Elution tests were performed according to the developed method after adding each $2 \mu \mathrm{g}$ of MCs to $1 \mathrm{ml}$ of hexane. Although the elution patterns obtained with the three adsorbents were almost the same, the numbers of low recovery (below 5\%) substances observed using silica-gel was the fewest (5) compared with Florsil and alumina (12 and 20, respectively). Lower recovery substances were again polar substances, such as amines, phenols and polar pesticides. From these results, the most suitable clean-up chromatography for sediments was the combination of silica-gel and acetone-hexane solution. The elution position of each compound by silica-gel chromatography is given in Table 1. Since some polar substances that eluted in the Fraction 3 showed low recoveries due to tailing, an increase in the volume of eluant of Fraction 3 may be necessary. In the present study, since we used a commercial product (Waters Sep-Pac VAC $2 \mathrm{~g}$ ) instead of an open column, some substances, such as 4-n-nonylphenol and $n$-alkanes (Table 1 ), were eluted from the cartridges, themselves; thus, when using commercial products, attention must be paid to the levels of substances eluted from the product in method blanks to allow for either background correction of sample data or the removal of such substances from contaminant reporting.

\section{Overall recovery tests}

In order to confirm the performance of the developed method, we conducted overall recovery tests. We added each $1 \mu \mathrm{g}$ of MCs to sediment samples ( $10 \mathrm{~g}$ wet wt) collected from the mouth of Dokai Bay in Kitakyushu City (total organic carbon, $0.4 \%$ ), and then treated the samples according to the developed method. Quantification was performed using the internal-standard method instead of the AIQS-DB measurement. Recoveries of the MCs were calculated after background subtraction, i.e. by subtracting the amounts detected in a non-spiked sample from the amounts detected in the spiked samples. Recoveries of $80 \%$ of the MCs were in the 60 to $120 \%$ range (Table 1). LLE and/or column chromatography seems to cause low recovery (below $60 \%$ of recovery). In the overall recovery tests, the recoveries of 21 out of the $119 \mathrm{MCs}$ were lower than $60 \%$; of these 21 compounds, 10 and 15 compounds also showed low recovery (below 60\%) in LLE and silica-gel column chromatography, respectively, which indicates that LLE and silica-gel column chromatography are the causes of their low recovery. Since benzidine is known to be oxidized during analytical procedures, ${ }^{12,13}$ some amines also seem to be oxidized, and show low recoveries. Except for these polar substances, $80 \%$ of MCs show good recovery; $70 \%$ of MCs showed 60 to $120 \%$ of recovery with a variation (repeatability) less than $20 \%$. Some substances, such as $n$-alkanes, octanol and 4-n-nonylphenol, had recovery $>100 \%$ due to the effects of the contamination from a silica-gel cartridge column. From the overall recovery test, it was confirmed that the developed 
comprehensive method can analyze most SVOCs in sediments except for polar substances. In addition, the results of the overall recovery test provide useful information for predicting the recoveries of substances registered in the database (Table S1). The information is as follows: (1) generally, the overall recovery decreases with decreasing $\log K_{\text {ow }}$, (2) substances whose $\log K_{\text {ow }}$ are below 1 cannot be analyzed quantitatively by the developed method, (3) if substances dissociate in water, even if their $\log K_{\text {ow }}$ values are larger than 1 (e.g. pentachlorophenol), their recoveries are below $60 \%$, (4) substances that have hydroxyl functional groups and/or amino functional groups show low recoveries, and substances that have a $\log K_{\text {ow }}$ below 3 and also have functional groups that contain active hydrogen cannot be recovered by more than $60 \%$, (5) the recovery decreases with the increase in the number of active hydrogen functional groups.

\section{Detection limit}

The AIQS-DB uses TIM to measure a large number of SVOCs, and in doing so sacrifices sensitivity. However, current GC-MS instruments have much better sensitivity compared with those of instruments manufactured one decade ago; they can measure mass spectra at pg levels. Detection limits (DL) for the target substances were estimated from concentration ratio (or, ratio of the dry weight of a sample to the volume of a final concentrate), and the instrument detection limit (IDL). For $83 \%$ of the chemicals in the AIQS-DB, the DL was $\leq 4 \mu \mathrm{g} / \mathrm{kg}$ dry wt. In addition, since the GC-MS used in this study can perform SIM/TIM measurement, we measured a sub-set of substances in the database, such as some PCBs, organochlorine pesticides, PAHs and sterols, by SIM. Since sensitivity by SIM was tentimes higher than by TIM, the DL of the PCBs, organochlorine pesticides and PAHs was $\leq 0.4 \mu \mathrm{g} / \mathrm{kg}$ dry wt.

\section{Analysis of standard reference material}

In order to validate the developed comprehensive method, we analyzed a standard reference material (SRM, NIST 1941b, Organics in Marine Sediment; National Institute of Standards and Technology, Gaithersburg, MD, USA). Instrumental measurements were done by TIM/SIM; PAHs were measured by TIM and quantified by the AIQS-DB and PCBs and organochlorine pesticides were measured by SIM and quantified by calibration curves in the AIQS-DB. Although the number of compounds certified in the SRM is much smaller than compounds registered in the AIQS-DB, if analytical results agree with certificated concentrations, analytical results for other compounds (except for highly polar substances) can be assumed be close to true concentrations. The results are given in Table S4 (Supporting Information). The means of the ratios of results to the certified values was from 32 to $175 \%$ (average: 90\%, RSD: 29\%). Although results for some of the substances differed from the certificated values, the concentrations determined for 33 out of 50 substances fell within $\pm 30 \%$ of the certified values, which indicates that although the accuracy of the comprehensive method is slightly lower than that of the conventional methods, it is sufficient for environmental surveys.

Confirmation of applicability and usefulness of the developed comprehensive method

In order to confirm that usefulness of the developed comprehensive method, we analyzed real sediments that were taken in from waterways in Vietnam. In this examination, we measured samples by GC-MS-SIM/TIM and identified and quantified the substances in the AIQS-DB. Recoveries of most of the surrogates (Table S2) were at the same level as those of overall recovery tests. However, recoveries of some polar substances were above $100 \%$. The cause of this excess recovery seems to be the matrix effect that is well known in food analysis. ${ }^{15}$ Since the sediments analyzed were heavily polluted, and the clean-up procedure in the developed method is simple, the amount of matrix in the final concentrates were enough to act as a liquid phase of a capillary column. Except for the matrix effect, it is confirmed that there was no problem in the individual sample analysis from reasonable surrogate recovery. The results of surrogate analysis show that the addition of surrogates before extraction is useful to evaluate the right or wrong of individual sample analysis. Although in this study 38 surrogates were used, the number of surrogates can be reduced to around 10 (e.g. those shown in Table S2); acid and basic substances, substances eluted in Fraction 3 and substances with a constantly high recovery.

One hundred-fourteen and 66 substances were found in the HCM sediments by TIM and SIM, respectively (Table S5, Supporting Information). Since Site 2 is in a small canal located in the center of HCM, its detected concentrations are much higher than at Site 1, the much larger Saigon River. Characteristic substances were chemicals that seem to be discharged from domestic sources: sterols, particularly coprostanol from mammalian feces, ${ }^{16}$ and nonylphenol resulting from degradation of common nonionic surfactants. ${ }^{17}$ Persistent organic pollutants (POPs), such as PCBs and organochlorine pesticides, which have been banned in Vietnam since the $1990 \mathrm{~s},{ }^{18}$ were also found at Site 2 at relatively high concentrations. In addition, chloropyrifos and permethrin 1 and 2 were detected at Site 2 . These results show that sediments in small canals in HCM are heavily polluted by chemicals discharged from domestic sources due to a lack of sewer system in HCM, and their effects reach to the main stem of a big river (Site 1). From the results obtained, a more holistic picture of organic pollution at sampling sites can be obtained. The evaluation results concerning the performance of the developed method clearly show that the comprehensive analysis is a very useful tool for rapid screening of environmental pollution, because it can provide a whole pollution picture that provides a lot of information, including the necessity of further detailed survey.

\section{Conclusions}

We have developed a comprehensive analytical method for SVOC in sediments using the AIQS-DB. From the recovery tests using $119 \mathrm{MCs}$, it was confirmed most SVOC, except for polar substances, can be analyzed quantitatively. From the analysis of SRM, the accuracy and precision of the developed method are slightly lower than that of conventional methods that were developed for targeted analysis. Although the sensitivity of AIQS-DB using TIM may be insufficient for some chemicals that usually require a high sensitivity, such as POPs, this weak point can be overcome by using SIM/TIM mode; in the present study we applied SIM to POPs in SRM and sediments in Vietnamese rivers and obtained good results. The method was applied to actual sediments in rivers in HCM. We found the pollution by domestic chemicals and POPs that had been banned to use in the 1990s. From the pattern of detected chemicals as well as their concentration patterns, we can obtain a more holistic pollution picture in rivers. From these results, it is confirmed that the developed comprehensive method using the AIQS-DB is a useful tool for the screening of pollution resulting from accidents and natural disasters because a large number of chemical substances can be measured rapidly at relatively low cost. In addition, the whole pollution picture, which is difficult 
to obtain by conventional methods, is useful to find emission sources in survey areas.

\section{Acknowledgements}

We thank Dr. Graeme Allinson (Department of Primary Industries, Queenscliff, Australia) and Dr. Mayumi Allinson (University of Melbourne, Melbourne, Australia) for their kind proofreading, useful comments and constructive suggestions on this manuscript.

\section{Supporting Information}

Table S1 is a list of organic compounds in AIQS-DB. Table S2 gives the recoveries of surrogate compounds. Table S3 shows the quantification and confirmation ions of SIM. Table S4 lists analytical results of a standard reference material (NIST 1941b, Organics in Marine Sediment). Table S5 gives analytical results of sediments in rivers in Ho Chi Minh, Vietnam. These materials are available free of charge on the Web at http://www.jsac.or.jp/ analsci/.

\section{References}

1. R. Binetti, F. M. Costamagna, and I. Marcello, Ann. Ist Super Sanita, 2008, 44, 13.

2. Environmental Quality Standards for Water, Ministry of the Environment, Japan, http://www.env.go.jp/en/standards/.

3. K. Kadokami, K. Tanada, K. Taneda, and K. Nakagawa, Bunseki Kagaku, 2004, 53, 581.

4. K. Kadokami, K. Tanada, K. Taneda, and K. Nakagawa, J. Chromatogr., A, 2005, 1089, 219.

5. T. Miyazaki, K. Kadakami, Y. Sonoda, D. Jinya, T.
Yamagami, K. Toubou, and H. Ogawa, Bunseki Kagaku, 2011, 60, 543.

6. D. Jinya, T. Iwamura, K. Kadokami, and T. Kusuda, $J$. Environ. Chem., 2011, 21, 35.

7. Environment Health Department, Ministry of the Environment, Japan, "Chemicals in the Environment", 1987 - 2002, Ministry of the Environment, Japan, Tokyo, Japan.

8. N. Tatarazako, M. Katoh, and K. Kadokami, in "Scrap Tire Derived Geomaterials-Opportunities and Challenges", ed. H. Hazarika and K. Yasuhara, 2008, Taylor \& Francis, London, UK, 109.

9. Method 3660B, "Sulfur Cleanup", 1996, US Environmental Protection Agency, Cincinnati, OH.

10. Y. Kawamura, M. Takeda, and M. Uchiyama, J. Food Hyg. Soc. Jpn., 1978, 64, 714.

11. Method 3545A, "Pressurized Fluid Extraction (PFE)", 2000, US Environmental Protection Agency, Cincinnati, $\mathrm{OH}$.

12. Method 8270D, “Semivolatile Organic Compounds by Gas Chromatography/Mass Spectrometry (GC/MS)", 2007, US Environmental Protection Agency, Cincinnati, OH.

13. "Standard Methods for the Examination of Water and Wastewater", ed. A. D. Eaton, L. S. Clesceri, E. W. Rice, and A. E. Greenberg, 21st ed., 2005, American Public Health Association, Washington, D.C., 6 - 66.

14. K. Kadokami, K. Sato, Y. Hanada, R. Shinohara, M. Koga, and H. Shiraishi, Anal. Sci., 1995, 11, 375.

15. K. Grob, "Split and Splitless Injection in Capillary Gas Chromatography", 3rd ed., 1993, Huthig Buch Verlag, Heidelberg, Germany, 362.

16. K. Ogura, Chikyu Kagaku, 1983, 17, 68.

17. W. Giger, P. H. Brunner, and C. Schaffner, Science, 1984, 225,623 .

18. D. Q. Hung and W. Thiemann, Chemosphere, 2002, 47, 357. 